\title{
MEDUSA: a Multi-Environment Dual-robot for Underwater Sample Acquisition
}

\author{
Diego Debruyn ${ }^{1}$, Raphael Zufferey ${ }^{1}$, Sophie F. Armanini ${ }^{1}$, Crystal Winston ${ }^{1}$, \\ André Farinha $^{1}$, Yufei Jin ${ }^{1}$ and Mirko Kovac ${ }^{1,2}$
}

\begin{abstract}
Aerial-aquatic robots possess the unique ability of operating in both air and water. However, this capability comes with tremendous challenges, such as communication incompatibility, increased airborne mass, potentially inefficient operation in each of the environments and manufacturing difficulties. Such robots, therefore, typically have small payloads and a limited operational envelope, often making their field usage impractical. We propose a novel robotic water sampling approach that combines the robust technologies of multirotors and underwater micro-vehicles into a single integrated tool usable for field operations. The proposed solution encompasses a multirotor capable of landing and floating on the water, and a tethered mobile underwater pod that can be deployed to depths of several meters. The pod is controlled remotely in three dimensions and transmits video feed and sensor data via the floating multirotor back to the user. The 'dual-robot' approach considerably simplifies robotic underwater monitoring, while also taking advantage of the fact that multirotors can travel long distances, fly over obstacles, carry payloads and manoeuvre through difficult terrain, while submersible robots are ideal for underwater sampling or manipulation. The presented system can perform challenging tasks which would otherwise require boats or submarines. The ability to collect aquatic images, samples and metrics will be invaluable for ecology and aquatic research, supporting our understanding of local climate in difficult-to-access environments [Video attachment: https://youtu.be/v4xWmEHUSM4].
\end{abstract}

Index Terms-Index TermsEnvironment Monitoring and Management Index TermsAerial Systems: Applications Index TermsMarine Robotics Index TermsField Robots Index TermsMultiRobot Systems Index TermsSoft Sensors and Actuators

\section{INTRODUCTION}

$\mathbf{M}$ ULTI-MODAL robots are able to move effectively in different media (water, ground, air) and transition between them. This remarkable capability, inspired by nature, allows for the advantages of different types of robots to be combined in a single platform. Thus, it becomes possible for the same robot to cover long distances and fly over obstacles, like an aerial vehicle, and move efficiently underwater for

Manuscript received: February 25, 2020; revised: May 5, 2019; accepted: May 28, 2020.

This paper was recommended for publication by Editor Youngjin Choi upon evaluation of the Associate Editor and Reviewers' comments. This work was funded by EPSRC (award no. EP/R009953/1, EP/L016230/1 and EP/R026173/1), NERC (award no. NE/R012229/1) and the EU H2020 AeroTwin project (grant ID 810321). Mirko Kovac is supported by the Royal Society Wolfson fellowship (RSWF/R1/18003).

${ }^{1}$ All the authors are with the Aerial Robotics Laboratory, Imperial College London, UK. m.kovac@imperial.ac.uk

${ }^{2}$ Mirko Kovac is also affiliated with the Materials and Technology Center of Robotics at the Swiss Federal Laboratories for Materials Science and Technology, Switzerland.

Digital Object Identifier (DOI): see top of this page.

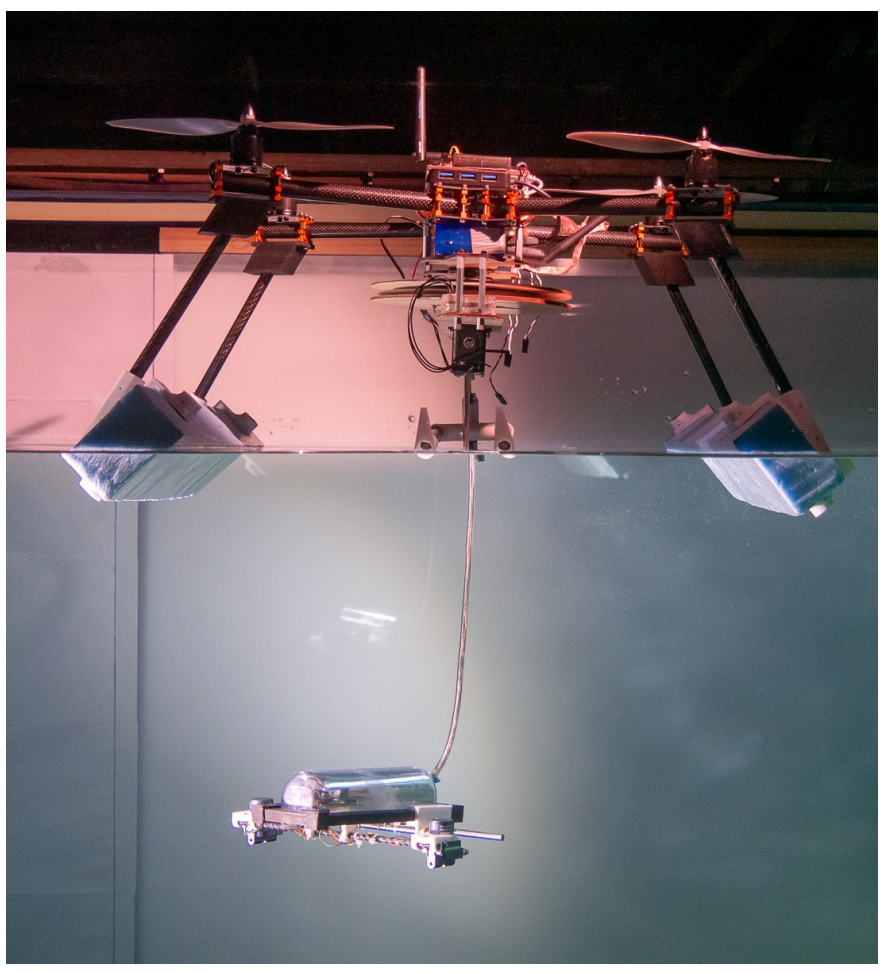

Figure 1. Photograph of the MEDUSA system: the multirotor robot is floating, while the pod has been partially uncoiled and is moving underwater.

long periods of time like a submersible. Aerial-aquatic robots, specifically, could vastly facilitate operations involving water sampling or underwater surveying, which currently rely on manpower (e.g. hand-dropped sensors, rope-access to spaces under oil rigs) or on the complex integration of different vehicles (e.g. ships carrying deployable submarine robots). Using a single multi-purpose vehicle for such tasks instead would lead to more effective data collection as well as improved safety and reduced costs. However, effective operation in and transitioning between - vastly different mediums (water and air) presents a considerable challenge, which continues to hinder the development of aerial-aquatic robots for real-world applications.

Most aerial-aquatic robots developed to date [1], [2] fall into the fixed-wing or rotating-wing category, or involve hybrid fixed/rotating-wing solutions [3], [4].

Fixed-wing vehicles [5], [6], [7], [2], [8], [9], [10] have the advantage of fast deployment and longer flight ranges, but both air-water transitioning and underwater locomotion 


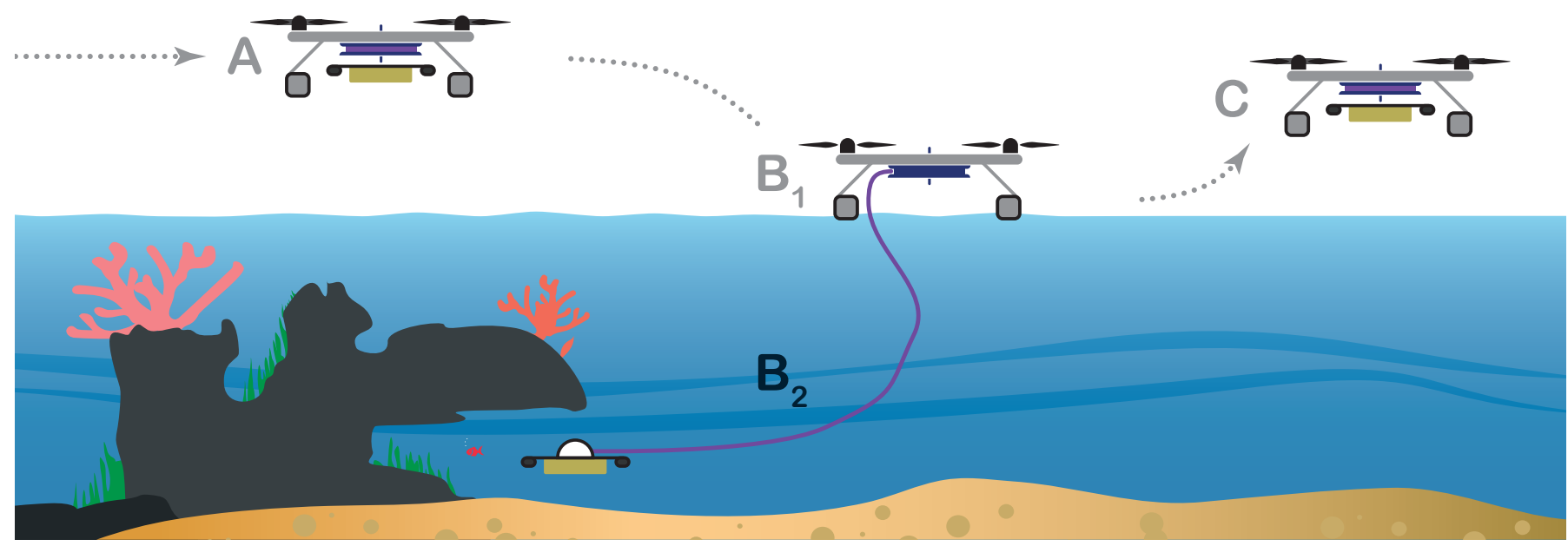

Figure 2. Schematic of the operation principle of the dual system. A. The flying module carries the pod (yellow) to set location. B1. The flying module lands on the water surface and floats due to buoyancy elements. B2. The coiler subsystem (blue) releases the tether and the pod moves vertically (buoyancy control) and horizontally (jet) to reach the target underwater location. Live video is transmitted and a sample taken. C. The tether is coiled again and the drone flies back to the user.

are challenging to achieve. Traditional fixed-wing take-off and landing on water requires relatively extensive areas of calm water, which are not always available. Furthermore, transitioning to or from a fully submerged state involves either high power density propulsion and impact-resistant structures - for impulsive transitions - or high-accuracy control - for slow transitions. Long-duration missions likewise present a challenge as most fixed-wing vehicles are optimised for flight and hence have limited underwater locomotion capabilities.

Multirotors, by contrast, can provide a simpler alternative, often made from off-the-shelf components. Due to their vertical take-off and landing capability, they can land on water and sink, without requiring added design features. Several multirotors have achieved a degree of aerial-aquatic mobility [11], [12], [13], however these vehicles have a short flight time and range when compared to fixed-wing solutions. While underwater propulsion can be achieved using the same propellers as for flight, this comes at a performance cost and does not always lead to effective locomotion.

Most existing work stops short of demonstrating a full mission cycle, focusing on specific operation phases or on simulations [14], [15]. This points to the high complexity of the proposed solutions, especially for long-duration underwater missions, and has prompted some research into simpler approaches. Rather than designing a single robot capable of operating efficiently in both environments, one alternative is to use a pair of robots inspired by the symbiotic relationships sometimes found in nature.

Many animals develop such relationships to overcome their natural morphological shortcomings. An example is the relationship between remoras and sharks. By attaching itself to a shark, the remora is able to travel long distances while being protected by the shark and expending only minimal amounts of energy. At the same time, the remora eats bacteria or parasites that have grown on the shark's skin, which helps the shark avoid infections [16]. In nature, symbiotic relationships develop out of a need for food, protection and/or hygiene.
While robots face different challenges, the same principle can be applied, where the respective strengths of two robots compensate for each other's weaknesses.

One solution that has been suggested, in a somewhat similar vein, is the use of low-flying quadrotors dropping rope-attached sensors into the water and then retracting them again [17]. This is a relatively simple approach but is constrained by the short battery time characteristic of quadrotors. Moreover, no control is provided over the positioning of the dropped sensors in the water.

In this paper, we propose a new solution for robotic water sampling, comprising a submersible pod tethered to a quadrotor. The quadrotor flies to a designated location, lands on the water surface and deploys a pod to collect measurements. Landing on the water surface, as opposed to hovering above it, allows for longer-duration missions when compared to existing aerial robot-based systems, as the quadrotor can float passively while the pod performs measurements. When compared to single-robot systems, the advantage of our system lies in its modularity and ease of development. The quadrotor is based on off-the-shelf components, so it does not need to be designed and manufactured from scratch, and remains outside the water, hence requires no waterproofing. Moreover, single-robot systems designed to collect data underwater still face challenges with demonstrating air-water transition, controlled flight and controlled underwater locomotion in a single system [18].

Our contributions include the following: (i) a novel approach for robotic water sampling, that is simpler and better suited for field testing than available methods, (ii) development of communications, electronics and control frameworks for a symbiotic dual-robot system, (iii) implementation of a new prototype (MEDUSA: Multi-Environment Dual-robot for Underwater Sample Acquisition, shown in Fig. 1) validating the proposed concept, and (iv) demonstration of the operation of the new system in both a lab environment and outdoor field tests. 


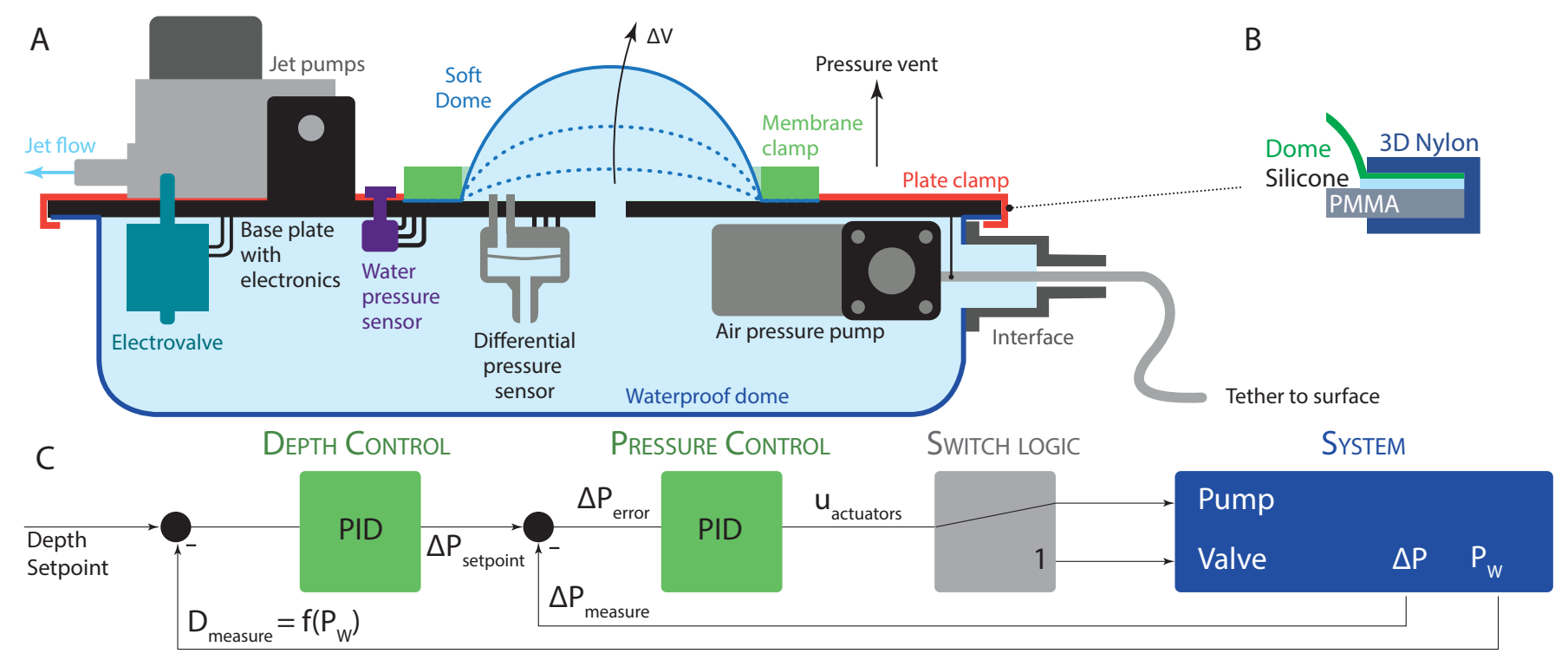

Figure 3. A. Side view of the underwater pod with its main internal components. B. Seal clamp structure. C. Depth control schematic.

\section{DESIGN}

\section{A. Operation Principle and Envelope}

The MEDUSA symbiotic robot system is specifically designed for water sampling in difficult-to-reach locations. As shown in Fig. 2, the system consists of a quadrotor that can fly to specific locations, land on the water and deploy a smaller robot which collects water measurements or samples. The smaller robot (hereafter 'underwater pod') has 3 controlled degrees of freedom. It controls its depth through inflation of a soft membrane, its forward motion via the thrust produced by two pumps on the outside of its body, and its orientation around the vertical axis via a thrust differential between the same pumps. This allows the system to travel successfully to different difficult to reach and potentially far apart locations, and to collect various water samples or measurements within a single mission. The pod is connected to the quadrotor by a 5 meters long tether, which constrains its operating envelope to a spherical cap with slightly under 5 meters radius.

A specific scenario where MEDUSA can operate and perform better than other aerial aquatic solutions is the monitoring of coral reefs. As these environments are normally close to shore, the short mission range inherent to using a quadrotor is not critical. Moreover, these environments are very unstructured and normally encountered at shallow depths. The fivemetre range of the tether is thus sufficient for the mission and the underwater pod can manoeuvre the intricate features of the reefs due to its small form factor and nimbleness. Thanks to the features mentioned, water samples can be obtained in the vicinity of relevant biomes that would otherwise not be possible to access remotely.

\section{B. Deployment Flying Vehicle}

The flying platform is a custom quadrotor of approximately $1 \mathrm{~kg}$ and a payload of 800-900 grams. The double 2D plate frame is well-suited to affix the bottom-mounted coiling system, as shown in Fig. 4.

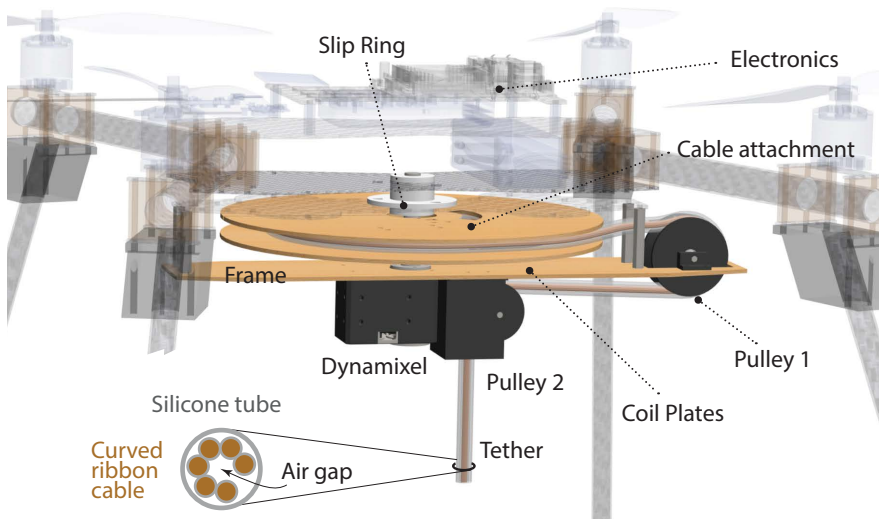

Figure 4. CAD view of the coiling mechanism installed at the base of the $\mathrm{X}$-frame quadrotor.

The underwater pod depends entirely on the tether for power, commands, relaying back data, live video and air supply. A design constraint for this system is its maximum depth, which has a direct impact on the required tether length. In order to have an operation envelope of a spherical cap of $5 \mathrm{~m}$ radius, an equivalent length of tether needs to be reliably coiled before flight and uncoiled once in the water. Furthermore, to minimise the impact of the tether on the pod motion, its stiffness needs to be reduced as much as the application permits and its buoyancy must be close to neutral. The developed custom tether, shown in Fig. 4 (bottom), consists of a $6 \mathrm{~mm}$ outer diameter silicone tube that shields the cable from sea-water and is bonded at its extremities. It contains a curved ribbon cable by Cicoil. Every strand is made from a twisted copper wire with silicone insulation, chosen with a $1 \mathrm{~A}$ specification. The ribbon cable leaves an air gap within the silicone tube that serves as a channel for air flow. The copper strands limit the minimum bending radius, permitting unrestricted air flow at all times. The end of the tube is sealed onto the air pump resting in the pod. 


\section{Underwater Pod}

The underwater pod consists of two external pumps, which steer the pod via differential thrust, and a main cabin, which houses the electronics, pump and membrane needed for vertical locomotion. This pump is sized to withstand pressure 5 meters underwater and determines the pod achievable depth. Inside the main cabin is a microcontroller, which handles communication between the quadrotor and the underwater pod. The cable that connects the underwater pod to the quadrotor via the coiling unit is used to send high-level commands to the pod. The actuators, sensors and microcontroller inside the pod are also powered by the quadrotor, with the wires connecting these systems to the quadrotor's battery also passing through the coiling mechanism. The main cabin is pressurised, reducing structural loads, therefore reducing weight and enhancing waterproofness.

The structure of the pod is shown in Fig. 3.A. Its dimensions are minimised for the smallest volume possible in order to reduce the amount of ballast needed to sink. For that purpose, the shape of the pod was designed to match the containing components. A semi-cylinder fits well and rests against a rigid support plate.

The PETG dome is vacuum-formed and has a lip to seal against the PMMA support plate as shown in Fig. 3.B. The silicone seal is made from Moldstar 15. It is maintained under pressure through a 3D-printed nylon clamp. The 4 C-Clamp segments fit against each other to seal the entirety of the perimeter. Rubber bands in the corners lock the clamps in place. This sealing method is reliable (more than 100 opening cycles), takes 10 seconds to seal and can be rapidly prototyped.

\section{SENSING AND CONTROL}

\section{A. Horizontal Actuation and Buoyancy Control}

The orientation of the underwater pod is controlled by two external jets which steer via thrust differential. When the floating quadrotor receives a command to turn the underwater pod, it adjusts the thrust generated by both of the external jets in order to rotate the pod. This is presently done via openloop commands, however a closed-loop control scheme could be easily implemented, if a specific orientation is required for a given mission.

The vertical locomotion of the underwater pod is controlled via buoyancy adjustments. This strategy is inspired by fish, who generally control their depth in the water by inflating or deflating a bladder inside their body, thus changing their buoyancy. Our underwater pod employs the same technique to control its buoyancy and resulting depth, using two pressure sensors and a pump. Once the pod receives a depth setpoint from the user, it uses a absolute pressure sensor to determine its current depth and a differential pressure sensor to determine the difference in pressure between the inside and outside of the cabin. The difference between current depth and desired depth is fed into a first control component ('depth control' block in Fig. 3.C), which determines the differential pressure required for the pod to attain and maintain the target depth. The computed pressure request is monitored to ensure that it remains within achievable bounds and does not cause the membrane to burst. A second PID controller ('pressure control') takes the difference between the measured differential pressure and the aforementioned required differential pressure as error signal, and computes the required actuator inputs. While a single signal is computed by the controller, the pod is controlled by the combination of a pressure pump and a valve. In a final step, the input to each actuator is therefore determined according to the following logic. If the actuator signal computed by the controller corresponds to an increased differential pressure demand, the signal is passed directly to the pump and the valve is closed, allowing pressure to build up. If, on the other hand, a decreased differential pressure is required, the pump is set to zero and the valve is opened to allow for pressure to be released.

\section{B. Sensor Payload}

MEDUSA is currently equipped with a camera and absolute pressure sensor, so that underwater images and their corresponding depths can be recorded. The camera is physically placed in the pod, it thus transmits underwater footage when the pod is deployed and aerial footage (fixed heading) when in flight. In future iterations, these sensors could be complemented by salinity, $\mathrm{pH}$, oxygen content, and other water quality sensors if the mission requires it. A custom water sampling device will also be developed.

\section{Electronics, Communication and Inter-System Tether}

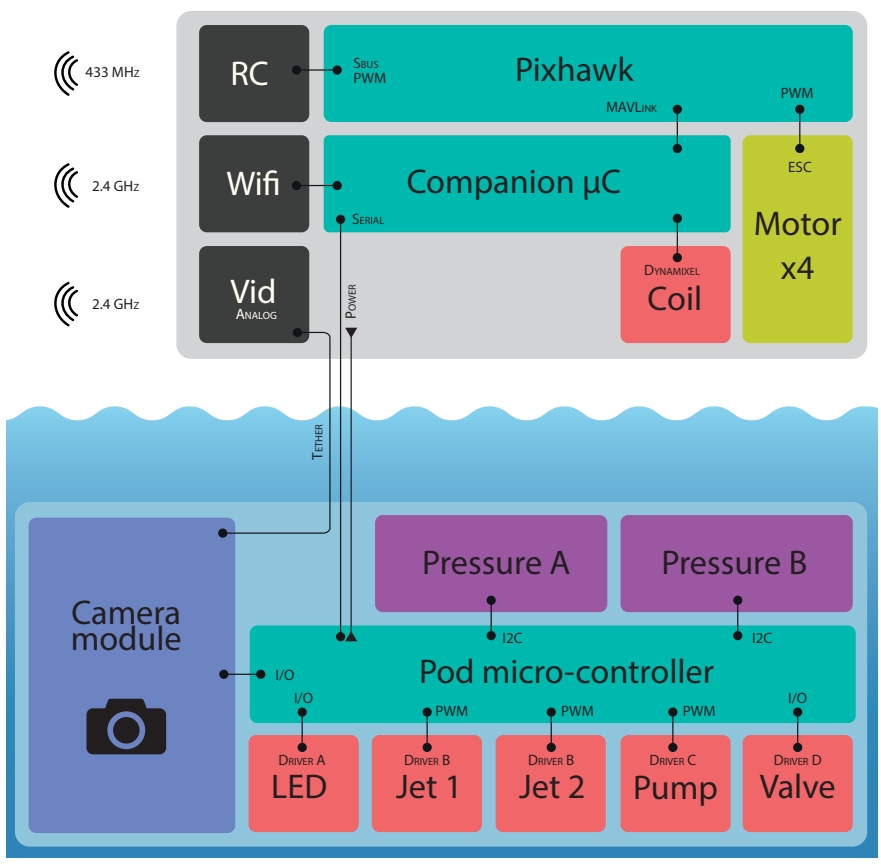

Figure 5. Communication and electronics framework showing both the drone subsystem (top) and the pod subsystem (bottom), linked by a custom tether.

The MEDUSA drone comprises a uniformed electrical network that spans from the flying vehicle to the underwater mobile pod (cf. Fig. 5). The companion computer runs ROS (Robotic Operating System), which handles high-level tasks and all the control of the system. It interfaces with the coiler 
subsystem, in charge of coiling and uncoiling the tether. The coiler is operated via a dynamixel servoactuator, running as a node in ROS. Two different serial links span from the companion microcontroller to the flight controller (MAVLINK, Micro Air Vehicle Link serial communication protocol) and to the underwater pod using ROSserial.

The flight controller handles the framework required for multirotor flight. It directly outputs the signals to the four Electronic Speed Control (ESC) board that drive the brushless motors. Additionally, the flight controller relays flight data to the companion computer via MAVLINK.

The end user interacts with the flight controller via a standard $433 \mathrm{MHz}$ radio link which provides kilometre-range operation. This connection transmits instructions and manual control commands and is supplemented by a telemetry link. The latter serves to send and receive commands that can change parameters and read system states. Analog video is broadcasted by radio and requires only one cable in the tether.

\section{RESUlts AND Discussion}

The developed system was tested both in a controlled laboratory setting, allowing for extensive underwater characterisation, and in realistic outdoor conditions. The experiments aimed to demonstrate the locomotion and manoeuvring capability of the system in both the horizontal and the vertical plane, the effectiveness of the depth control system, and the overall functioning of the robot in realistic outdoor conditions. Furthermore, preliminary tests were performed to assess the efficacy of the membrane-based buoyancy control method proposed, and a model was developed to yield more detailed insight into the behaviour of the membrane. The remainder of this section provides an overview of the main results obtained.

\section{A. Membrane-Based Actuation}

As elucidated in Section III-A, the vertical motion of the underwater pod is controlled through pressure-based inflation and deflation of a soft membrane (as shown in Fig. 6.B). An important preliminary step is to establish whether this type of actuation mechanism is adequate for stable depth control. To evaluate this, we fed the pod a number of differential pressure setpoints and observed the resulting changes in differential pressure and volume of the deforming membrane. This is equivalent to testing the system with only the inner loop 'pressure control' component of Fig. 3.C active. The aforementioned experiments were conducted outside the water with the pod at rest, so as to provide a clear view of the inflating membrane. The volume of the membrane at each time step was computed based on video footage. The inflated membrane is approximated as a spherical cap, whose volume can be computed from its base width $2 a$ and maximum height $h$ via the expression,

$$
V=\frac{1}{6} \pi h\left(3 a^{2}+h^{2}\right)
$$

Subsequent time synchronisation with the concurrent sensor measurements provided the corresponding differential pressure in the pod. As anticipated, and clarified by Fig. 7, the volume
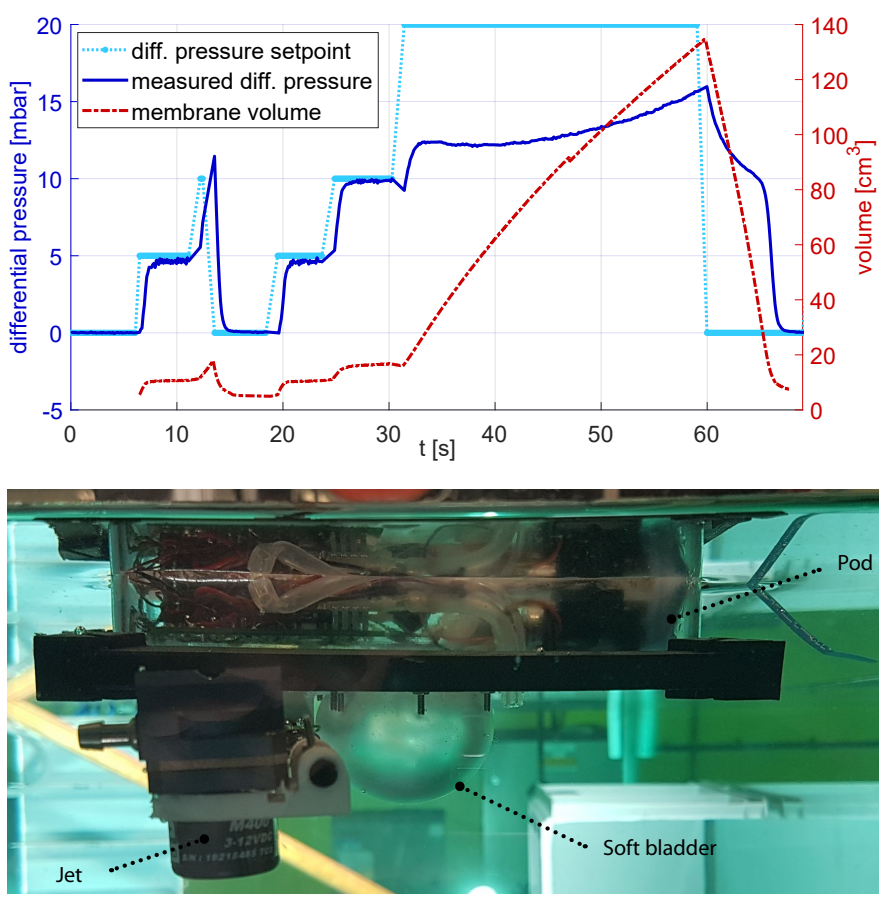

Figure 6. Evaluation of the buoyancy-based actuation mechanism for vertical motion. A. Measured membrane volume versus differential pressure for different pressure setpoints. B. View of the inflated membrane during experiments.

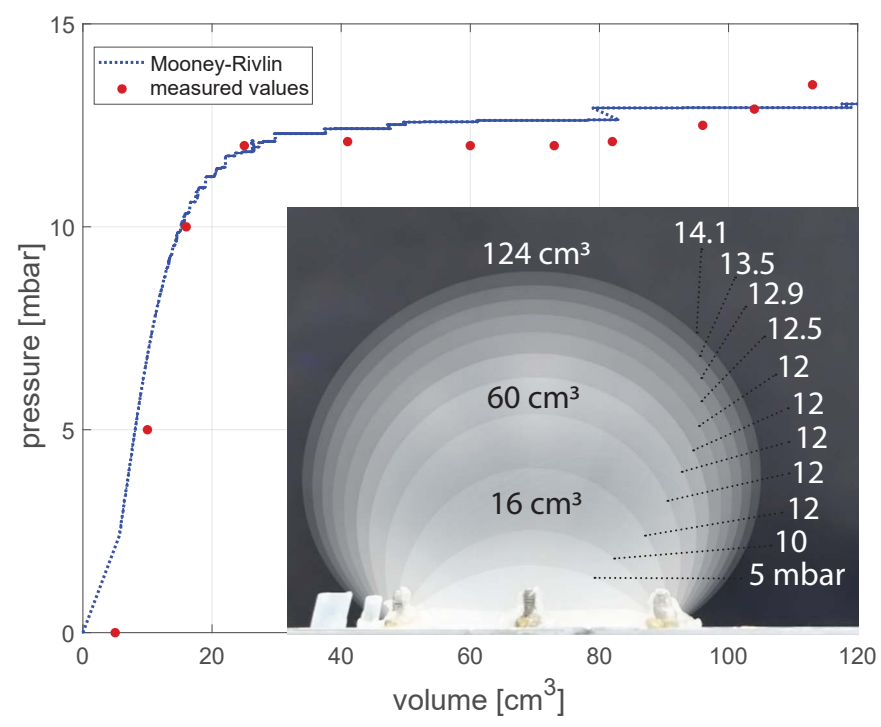

Figure 7. Membrane volume versus pressure: model-predicted and tracking data-reconstructed results.

of the soft membrane increases as pressure is increased. The two variables are approximately proportional up to a differential pressure of $10 \mathrm{mbar}$, after which point the relation between the two becomes significantly nonlinear, with a strong increase of volume at nearly constant pressure. It can also be seen that there is a limit to what differential pressures can be reached. Based on both observations, it was decided to limit the differential pressure to values below $10 \mathrm{mbar}$, where the membrane is not in danger of bursting and the relation between pressure and volume is approximately proportional. 

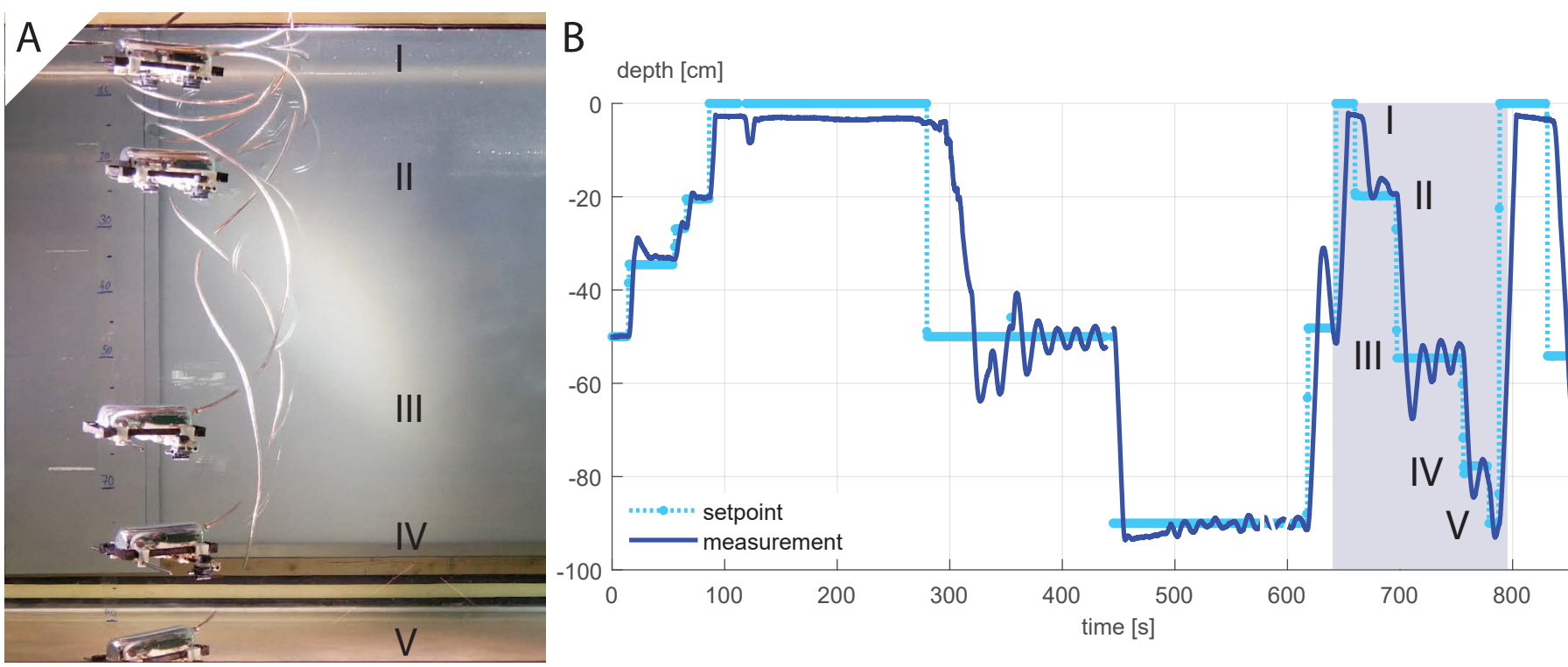

Figure 8. Vertical actuation and depth control results, showing the pod tracking a number of different successive depth setpoints.

Within this region, the pod can be rapidly stabilised at any given pressure (cf. Fig. 6.A).
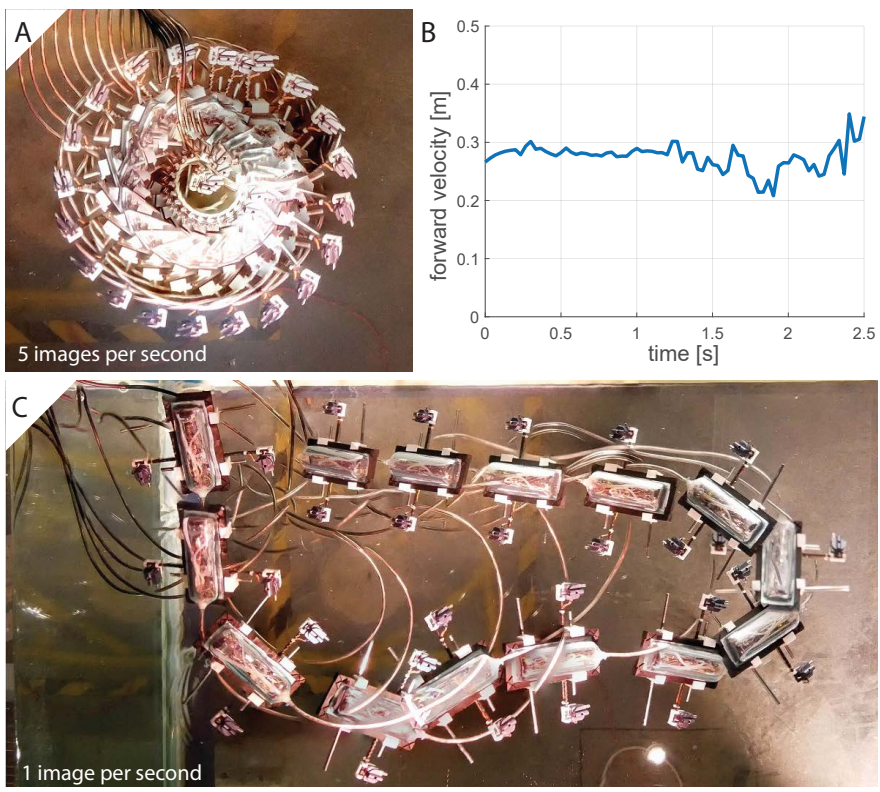

Figure 9. A. Composition showing maximum rotational control of the robot at $100 \%$ s. B. Manual control of the horizontal motion of the robot moving forward, turning and returning to base. C. Maximum velocity of the robot with jet thrusters at $100 \%$.

In order to further understand the behaviour of the membrane, it was modelled as a thin, hyperelastic, incompressible shell using the formulation developed by Adkins and Rivlin [19]. The membrane is described using a cylindrical coordinate system $(r, \Theta, z)$, where each particle on the surface of the membrane exists at a point $(\rho, \theta, \eta)$. The stretch ratios in the meridional and circumferential directions are $\lambda_{1}$ and $\lambda_{2}$, respectively.

$$
\begin{gathered}
\lambda_{1}=\sqrt{\frac{\left(\frac{\partial \rho}{d r}\right)^{2}+\left(\frac{\partial \eta}{\partial r}\right)^{2}}{1+\left(\frac{\partial z}{\partial r}\right)^{2}}} \\
\lambda_{2}=\frac{\rho}{r}
\end{gathered}
$$

The equilibrium equations of the deformed membrane yield

$$
\begin{gathered}
\frac{\partial T_{1}}{\partial \rho}+\frac{1}{\rho}\left(T_{1}-T_{2}\right)=P_{t} \\
K_{1} T_{1}+K_{2} T_{1}=P_{n}
\end{gathered}
$$

where $T_{1}$ and $T_{2}$ represent the meridional and circumferential stresses, $P_{n}$ represents the stresses normal to the membrane surface and $P_{t}$ represents pressure in the transverse direction. For this problem $P_{t}=0$. Finally, assuming that this is an incompressible material that can be modelled with a two term Mooney-Rivlin constitutive relation, we get

$$
\begin{aligned}
& T_{1}=2 C_{1} h\left(\frac{\lambda_{1}}{\lambda_{2}}-\frac{1}{\lambda_{1}^{3} \lambda_{2}^{3}}\right)\left(1+\alpha \lambda_{2}^{2}\right) \\
& T_{2}=2 C_{1} h\left(\frac{\lambda_{2}}{\lambda_{1}}-\frac{1}{\lambda_{1}^{3} \lambda_{2}^{3}}\right)\left(1+\alpha \lambda_{1}^{2}\right)
\end{aligned}
$$

where $C_{1}$ and $\alpha$ are constants that come from the constitutive relationship. This relationship is usually written in terms of $C_{1}$ and $C_{2}$ where $\alpha=C_{1} / C_{2}$.

The above equations were simplified into three first-order ODEs using the method described by Yang and Feng [20] and then solved using a Runge-Kutta algorithm, implemented as in Ref. [21]. Solving these ODEs yields the height of the inflated membrane bubble, which can then be used to calculate the volume as per eq. 1 . The material properties, $C_{1}$ and $\alpha$, could not be found for our particular material (Ecoflex 0020 ), so values for a similar material were chosen as a starting 
point, and they were then adjusted to fit our data [22]. The values used to describe the geometry of the membrane and the corresponding material constants that came from fitting the model to the data are given below.

Table I

MODEL PARAMETER VALUES.

\begin{tabular}{lccc}
$\mathbf{C}_{\mathbf{1}}[\mathbf{M P a}]$ & $\alpha$ & Thickness $[\mathbf{m m}]$ & Radius [mm] \\
\hline 0.0039 & 0.1 & 1.1 & 20 \\
\hline
\end{tabular}

\section{B. Vertical Locomotion and Control}

The first set of indoor tests was performed in a $1 \mathrm{~m}$ deep water tank, in order to evaluate the effectiveness of the depth control scheme. These experiments involved placing the pod in the water, and successively sending it different depth setpoints to track, while always remaining attached to the quadrotor via the tether. An example of the obtained results is illustrated in Fig. 8, where it can be observed that the pod is able to track user-defined depths effectively, and that the developed buoyancy-based actuation can be used for vertical manoeuvring. New setpoints are consistently reached within approximately $5 s-$ a short time, given the relatively slow motion of the pod in the water.

Further it was found that, especially when moving downwards (to greater depths), the pod develops a slow oscillatory movement around the target depth. This may indicate that the actuation mechanism is unable to fully cancel out all oscillatory motion. Nonetheless, the observed fluctuations typically have a magnitude of approximately $2-5 \mathrm{~cm}$. Considering the intended domain of operation in the range of several metres, as well as the non-ideal experimental conditions, which inevitably involved some water movement, the attained accuracy of the system can be considered substantial despite the aforementioned oscillations. Note that, by contrast, when moving to smaller depths, the robot appears to stabilise more accurately and rapidly. This difference in behaviour is likely due to the combined pump and valve-based actuation explained in Sec. III-A, according to which increases in depth are obtained through valve opening.

\section{Horizontal Locomotion}

While large horizontal distances are covered by displacing the quadrotor itself, and horizontal movement of the pod can hence be limited in range, it is an important asset that the pod is able to manoeuvre and position itself within the region of interest. It may for instance be necessary for a particular mission to approach underwater objects, ranging from oil platform risers to coral reefs, or even to move underneath or inside a structure such as a pipe. In this context, the lateral manoeuvrability and performance of MEDUSA was tested.

Results show that the jet-based actuation provides sufficient thrust, leading to a manoeuvrability well-suited to the types of missions envisaged for the robot. As shown in Fig. 9 (left), the pod can reorient itself rapidly and perform horizontal turns at up to $100 \%$ s, as well as reach given target locations within the range allowed by the tether, reaching a speed of up to approximately $0.35 \mathrm{~m} \mathrm{~s}^{-1}$ (cf. Fig. 9).

While the actuation mechanism does not provide direct control over all degrees of freedom, the combination of vertical and horizontal actuation leads to a comprehensive range of motion. By manoeuvring the pod into a closed space with a single lateral opening (cf. Fig. 10 left), we for instance showed that narrow confined spaces can be reached, which is an important capability for the type of system proposed.

\section{Outdoor Testing}

Outdoor field tests were performed on a lake, surrounded by dense forest (cf. Fig. 10). The water conditions were calm, but there were gusty winds. One of the most critical aspects of an aerial-aquatic robot is the transition from air to water and vice-versa. We successfully demonstrate multiple landings and take-offs from the water of the quadrotor carrying the coiling unit and pod. The downwash of the propellers does not create significant spray during take-off and in general no significant difficulties were encountered. The tests suggest that the robotic concept can function reliably and robustly in realistic and challenging conditions, similar to those that would be encountered in a typical application scenario. Underwater operation of the pod is not shown in the reported field tests due to the low visibility in the pond (less than $10 \mathrm{~cm}$ ). This would have hindered any visual observation of the position of the pod or any relevant remote video recording.

While the tether is a vital element, containing electronic and pneumatic links and ensuring that the pod can always be recovered, it also limits the operating range of the system and may tangle or break. In general, MEDUSA is designed to operate in mild wind and water conditions, where the quadrotor can float in an approximately steady position. The envisaged applications would take place in favourable weather or in calm waters, e.g. on lakes.

\section{CONCLUSIONS}

This article presents a novel concept for automated water sampling in difficult-to-reach locations, based on a dualrobot system consisting of a multirotor that can reach remote locations rapidly and land on the water, and an underwater pod that can stream live video and take samples. The proposed solution significantly reduces the limitations and difficulties typical of single-body aerial-aquatic robots, while still benefiting from the same advantages. A prototype was developed to demonstrate the approach. The design trade-off and the implementation and manufacturing methods required to build and operate such a system are discussed in the paper. Indoor and outdoor tests showed that the approach can be applied successfully in realistic conditions, and that the soft membranebased depth controller and jet-based horizontal actuation allow for accurate positioning and manoeuvring of the pod. The proposed approach allows for challenging underwater missions to be performed in a simple and robust way, which makes the method amenable for real-life applications.

Future work will further improve the field-readiness and reliability of the system. A structural revision of the pod, 

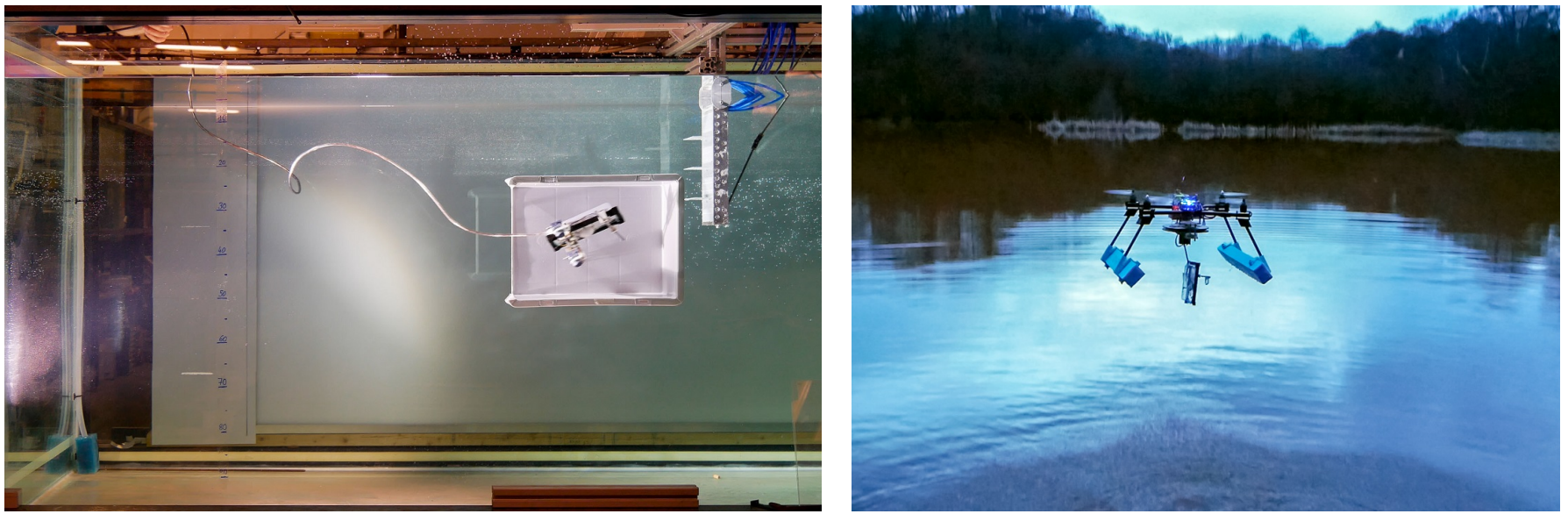

Figure 10. (Left) The robot is remotely manoeuvred into a confined space with a side opening of $30 \mathrm{~cm}$ (Right) The flying dual-robot platform shown during a take-off from water returning to the operator. Location: Silwood Park Campus, Imperial College London.

currently manufactured from vacuum-formed shells and 3Dprinted parts, will permit faster assembly, more reliable waterproofing and increased compactness. The geometry of the pod can also be optimised for improved hydrodynamics and stability. Additionally, further work will be undertaken to render the system fully autonomous. This will include implementing a position controller for the horizontal plane and a vision-based navigation component allowing more elaborate underwater mission execution. Some form of stabilisation in response to water currents would also be beneficial. Future field missions will be undertaken in clear waters.

\section{ACKNOWLEDGEMENTS}

The Multi-Terrain Aerial Robotics Arena is supported through a philantropic gift by Brahmal Vasudevan. The authors also thank the Imperial College London Department of Civil Engineering for usage of the hydrodynamics laboratory testing facilities.

\section{REFERENCES}

[1] K. H. Low, T. Hu, S. Mohammed, J. Tangorra, and M. Kovac, "Perspectives on biologically inspired hybrid and multi-modal locomotion," Bioinspiration \& Biomimetics, vol. 10, no. 2, 2015.

[2] R. Siddall and M. Kovač, "Launching the AquaMAV: Bioinspired design for aerial-aquatic robotic platforms," Bioinspiration and Biomimetics, vol. 9, no. 3, 2014 .

[3] D. Lu, C. Xiong, Z. Zeng, and L. Lian, "A multimodal aerial underwater vehicle with extended endurance and capabilities," in Int. Conf. Robotics and Automation (ICRA), 2019.

[4] J. Bian and J. Xiang, "Quuv: A quadrotor-like unmanned underwater vehicle with thrusts configured as x shape," Applied Ocean Research, vol. 78, 2018.

[5] R. Peloquin, D. Thibault, and A. L. Desbiens, "Design of a passive vertical takeoff and landing aquatic UAV," IEEE Robotics and Automation Letters, vol. 2, no. 2, pp. 381-388, April 2017.

[6] J. Liang, Y. Xing, W. Tianmiao, Y. Guocai, and W. Zhao, "Design and experiment of a bionic gannet for plunge-diving," Journal of Bionic Engineering, vol. 10, no. 3, 2013.

[7] J. Moore, A. Fein, and W. Setzler, "Design and analysis of a fixedwing unmanned aerial-aquatic vehicle," in 2018 IEEE International Conference on Robotics and Automation (ICRA), 2017.

[8] W. Stewart, W. Weisler, M. MacLeod, T. Powers, A. Defreitas, A. Gritter, M. Anderson, K. Peters, A. Gopalarathnam, and M. Bryant, "Design and demonstration of a seabird-inspired fixed-wing hybrid uav-uuv system," Bioinspiration \& Biomimetics, 2018
[9] R. Zufferey, A. Ortega, C. Raposo, S. F. Armanini, A. Farinha, R. Siddall, I. Berasaluce, H. Zhu, and M. Kovac, "SailMAV: design and implementation of a novel multi-modal flying sailing robot," Robotics and Automation Letters, vol. 4, no. 3, 2019.

[10] R. Zufferey, A. Ortega, A. Farinha, R. Siddall, S. F. Armanini, B. R. K. G. Nasr, M, and M. Kovac, "Consecutive aquatic jump-gliding with water-reactive fuel," Science Robotics, vol. 4, no. 34, 2019.

[11] H. Alzu'bi, I. Mansour, and O. Rawashdeh, "Loon copter implementation of a hybrid unmanned aquatic-aerial quadcopter with active buoyancy control," Journal of Field Robotics, 2017.

[12] M. M. Maia, D. A. Mercado, and F. J. Diez, "Design and implementation of multirotor aerial-underwater vehicles with experimental results," in 2017 IEEE/RSJ International Conference on Intelligent Robots and Systems (IROS), 2017, pp. 961-966.

[13] Z. Ma, J. Feng, and J. Yang, "Research on vertical air-water transmedia control of hybrid unmanned aerial underwater vehicles based on adaptive sliding mode dynamical surface control," Int. J. Advanced Robotic Systems, vol. 15, no. 2, 2018.

[14] P. J. Drews, A. Alves Neto, and C. M. F. M., "Hybrid unmanned aerial underwater vehicle modeling and simulation," in IEEE/RSJ Int. Conf on Intelligent Robots and Systems, 2014.

[15] S. F. Armanini, R. J. D. Siddall, and M. Kovac, "Modelling and simulation of a bioinspired aquatic micro aerial vehicle," in AIAA Modeling and Simulation Technologies Conf., Dallas TX, 2019, AIAA Paper 2019-3115.

[16] P. Kyne, “Occurrence of a sharksucker ('echeneis naucrates') on a northern river shark ('glyphis garricki') in a tidal riverine habitat," Northern Territory Naturalist, vol. 26, p. 21-26, 2015.

[17] J.-P. Ore, S. Elbaum, A. Burgin, and C. Detweiler, "Autonomous aerial water sampling," Journal of Field Robotics, vol. 32, no. 8, pp. 1095-1113, 2015. [Online]. Available: https://onlinelibrary.wiley.com/ doi/abs/10.1002/rob.21591

[18] R. Zufferey, S. F. Armanini, A. Farinha, and M. Kovac, "Adaptive morphology in aerial-aquatic robots," in Adaptive Motion in Animals and Machines (AMAM) Conf., 2019.

[19] J. E. Adkins and R. S. Rivlin, "Large elastic deformations of isotropic materials ix. the deformation of thin shells," Philosophical Transactions of the Royal Society, vol. 224, p. 505-531, 1952.

[20] W. H. Yang and W. W. Feng, "On axisymmetrical deformations of nonlinear membranes," ASME Journal of Applied Mechanics, vol. 37, p. 1002-1011, 1970.

[21] D. D. Washington, "Efficient methods for analyzing thin membranes subjected to transverse pressure and undergoing large deformation," Ph.D. dissertation, 2004.

[22] Y.-Y. Hsu, K. Lucas, D. Davis, B. Elolampi, R. Ghaffari, C. Rafferty, and K. Dowling, "Novel strain relief design for multilayer thin film stretchable interconnects," IEEE Transactions on Electron Devices, 2013. 